\title{
Code of Ethics for Community Informatics Researchers
}

\author{
Udo Averweg \\ Information Services, eThekwini Municipality and University of KwaZulu-Natal, Durban, South Africa \\ averwegu@durban.gov.za \\ Susan O'Donnell \\ National Research Council, Institute for Information Technology and University of New Brunswick, Fredericton, Canada \\ Susan.ODonnell@nrc-cnrc.gc.ca
}

\section{Preamble}

This draft Code of Ethics for Community Informatics (CI) researchers is intended to evolve. We invite CI researchers to contribute their ideas and perspectives based on their experiences of doing CI research and as new ethical concerns emerge.

The need for a Code of Ethics for CI researchers was identified at The $2^{\text {nd }}$ annual conference of the Community Informatics Research Network (CIRN2005) held in Cape Town, South Africa during 23-26 August 2005. Following this conference, the lead author undertook to formulate a Code of Ethics for CI researchers. The current document grew from an intensive collaborative iterative process with the second author. This document is now opened for general comment and feedback. This Code of Ethics for CI researchers is intended to be an evolving document - additional CI researchers may wish to contribute their perspectives and new ethical concerns may emerge in future.

Many countries have codes of research ethics and formal research ethics review boards in place for research involving humans, including research involving community participants. In these countries, researchers cannot proceed with their research until their research plans or protocols have been approved by an ethics review board. In many other countries, codes of research ethics are in development. CI researchers have a particular responsibility to conduct ethical research because the aim of CI research in general is to assist communities - often marginalized communities - to reach their development goals.

\section{Process for contributing to this draft}

The open journal system that publishes the Journal of Community Informatics (JoCI) has a built-in commenting system allowing readers to post comments below this article. We invite readers to contribute their energies to this drafting process by posting their comments on the system.

\section{Questions to get started}

a. Can you describe an ethical issue you experienced in your own CI research and does this draft Code of Ethics offer useful guidelines to address this issue?

b. Is it desirable to have an international Code of Ethics (such as this one) rather than national or community Codes of Ethics to address research in its local or national contexts?

c. Can you identify sections or points in the draft Code of Ethics below that need to be revisited and revised? Do you have specific suggestions for revision?

\section{Aims}

The aims of this document are to:

- develop a voluntary code of practice for CI researchers;

- contribute to the maintenance of high standards in CI research; and

- contribute to the broader ethical and professional debates within the CI profession. 


\section{Ethical Guidelines to be addressed in practice by CI researchers}

Guidelines or "codes of practice" offer a framework for the work of CI researchers. Making ethical decisions involves addressing a series of dilemmas: decisions have to be reached by the CI researchers involved and ethical guidelines enable these decisions to be made from an informed position. An "ethical conscience" is needed so that making ethical decisions become an automatic part of the CI research process.

The following are proposed as the minimum acceptable ethical standards of conduct for CI researchers:

a) The Objectives of CI Research

- The objective of CI, as with other research, is the formulation of new knowledge or insight into the means to enable and empower communities through the use of Information and Communications Technologies;

- the overall research aims of any CI study should be to benefit society and minimise social harm;

- the specific objectives of any research study should be negotiated with the subject communities so as to include both the objectives of the community and of the CI researchers; and

- this negotiation should include issues such as appropriate (and inappropriate) methods, rules for research conduct, ownership of data and the means by which this data may be disseminated and under what conditions.

b) Respect for human dignity

- CI research should aspire to protect the multiple interests of the person and the community - from bodily to psychological to cultural integrity;

- decisions by community members and others in respect of participation in research must be made from an informed position;

- research participants and communities must be protected from undue intrusion, distress, indignity, physical discomfort, personal embarrassment, and psychological or other harm; and

- research must be conducted with respect for all within the community and for all communities in society regardless of race, ethnicity, culture and/or religion.

\section{c) Using a subject-centred perspective}

- CI research entails an active involvement by research participants and ensures both that their interests are central to the project or study and that they will not be treated simply as objects;

- a subject-centred approach should recognise that researchers and research participants may not always see the harms and benefits of a research project in the same way;

- the CI researcher should endeavour to ensure that participation in research is voluntary;

- research must be conducted with respect for under-represented social communities and with attempts being made to avoid their exclusion and/or marginalisation; and

- the CI researcher should endeavour to ensure that subject-centred research is conducted with respect for and awareness of gender differences.

d) Respecting free and informed consent

- Research participants must be assured of and enabled to achieve the capacity and right to make free and informed decisions;

- research should commence only if prospective participants have been given the opportunity to give free and informed consent about participation, this free and informed consent has been given and if and as it is maintained throughout their participation in the research; and

- evidence of free and informed consent should ordinarily be obtained in writing. Where written consent is culturally unacceptable or where there are good reasons for not recording such consent in writing, the procedures used to seek free and informed consent should be documented.

e) Respecting vulnerable persons, justice and inclusiveness 
- Participants whose diminished competence or decision-making capacity make them vulnerable - such as children, institutionalised persons or others who are vulnerable - are entitled to special protection against abuse, exploitation or discrimination;

- no segment of the population should be unfairly burdened with the harms of research;

- research should neither neglect nor discriminate against individuals and groups who may benefit from advances in research;

- the CI researcher must endeavour to balance professional integrity with respect for national and international law;

- the concerns of relevant stakeholders and user groups must be adequately addressed; and

- the CI researcher should reflect on the consequences of research engagement for all participants and attempt to alleviate potential disadvantages to participation for any individual, category of person or community.

f) Respecting privacy and confidentiality

- Standards of privacy and confidentiality should protect the access, control and dissemination of personal information; and

- information that is disclosed in the context of a professional or research relationship must be held confidential.

g) Balancing harm and benefits

- The foreseeable harms of research should not outweigh anticipated benefits;

- research participants must not be subjected to unnecessary risks of harm;

- CI research is intended to produce benefits for the participants themselves, for other individuals or for society as a whole or for the advancement of knowledge;

- CI researchers have a duty to maximise net benefits to the participating community; and

- the CI researcher should reflect on the consequences of research engagement for all participants and attempt to alleviate potential disadvantages arising from participation for any individual, category of person or community.

h) Ensuring accuracy, appropriate use, ownership of data and research results

- the CI researcher must endeavour to ensure factual accuracy and avoid falsification, fabrication, suppression or misrepresentation of data;

- all data must be treated with appropriate levels of confidentiality and anonymity;

- ownership of the information that results from the CI research shall vest jointly with the community participating in the research and with the researcher;

- the anticipated use of such data should be made explicit in advance of the research being conducted and should be a part of the research protocol negotiated with the community and any change in such use should be negotiated with the community participating in the research;

- reporting and dissemination by the CI researcher must be carried out in a responsible manner; and

- the research results should be communicated to the community in a way that meets their needs.

i) Using appropriate research methodology

- Appropriate research methods must be selected on the basis of informed professional expertise; and

- methodology and findings must be open for full discussion and peer review.

\section{j) Research anchors}

- The research process should not involve any unwarranted material gain or loss for any participant;

- any debts to previous research as a source of knowledge, data, concepts and methodology must be fully acknowledged in all outputs;

- the CI researcher will not seek personal advantage to the detriment of the CI community; and 
- the CI researcher will accept full responsibility for any research to be undertaken and deliver what the researcher purports to deliver.

\section{Ethical guidelines for research involving Aboriginal communities}

- The CI researcher must understand the cultural responsibilities that accompany traditional knowledge or sacred knowledge and strictly comply with community expectations and protocols in possessing such knowledge;

- community jurisdiction over the conduct of research must be understood and respected;

- communities must be given the option of a participatory research approach;

- CI researchers must fully inform the community leadership or appropriate authorities and obtain prior approval from the community leadership or other appropriate authorities before research can be conducted in the community;

- confidentiality concerns of the community must be respected and addressed;

- CI researchers should recognise that the principle of individual autonomy may be reinterpreted in culturally specific ways that defer individuality to the interests of the Aboriginal community as a whole;

- inclusion of cultural knowledge in research must be under mutually agreed terms and with the guidance of the knowledge holders in the community;

- Aboriginal peoples and their respective communities retain rights to their knowledge, cultural practices and traditions that are shared with the researcher(s). It is the responsibility of the CI researcher to support mechanisms for protection of cultural knowledge that is shared during the research;

- research must be of mutual benefit to the community and researchers;

- CI researchers should support the development of education, research and training (including training in research ethics) for Aboriginal peoples and communities;

- CI researchers have an obligation to learn about and apply Aboriginal cultural protocols relevant to the particular Aboriginal community;

- CI researchers should make the best effort to translate publications or reports into the language of the community;

- CI researchers should ensure that there is effective on-going communication in a manner that is accessible and understandable to the community;

- Aboriginal communities have rights to control and determine their proprietary interests in the collection, use, storage and potential future use of data;

- all Aboriginal communities have a right to participate in the interpretation of data and/or review of conclusions drawn from the research to ensure accuracy and sensitivity of interpretation; and

- community members have the right to due credit and participation in dissemination of results and publications must recognise the contribution of the community where appropriate and in keeping with confidentiality agreements. It is the discretion of the community partners as to how their contributions will be acknowledged.

\section{Ethical considerations for researchers in different countries}

- Canada: Tri-Council Statement (TCPS) on Ethical Conduct for Research Involving Humans http://pre.ethics.gc.ca/

\section{Acknowledgement}

Excerpts made from "An EU Code of Ethics for Socio-Economic Research" (2004), IES Report 412 by The Institute for Employment Studies, Brighton, United Kingdom, the Tri-Council Statement (TCPS) on Ethical Conduct for Research Involving Humans, Canada and the Canadian Institutes of Health Research Guidelines for Health Research Involving Aboriginal Peoples. 